\title{
A NEURAL NETWORKS DECISION-SUPPORT SYSTEM FOR BIDDING IN CONSTRUCTION
}

\author{
Wanous, M., Boussabaine, A.H. and Lewis, J. \\ School of Architecture and Building Engineering, Liverpool University, UK \\ mohamad@liverpool.ac.uk
}

\begin{abstract}
One of the various strategic decisions that have to made by construction contractors is whether or not to submit a bid for a new project when an invitation has been received. An innovative neural network model is proposed in this paper to help contractors in making their bid/ no bid decisions. This model is based on one hundred and sixty two real bidding situations. The model was tested on another twenty real cases. $90 \%$ of the actual decisions of the testing sample were successfully predicted, which suggests that the model is very reliable and the ANN technique is suitable for modelling the bid/ no bid decision.
\end{abstract}

Keywords: ANN, ANN Bidding Model, "Bid/ No bid" Criteria, Construction, Syria.

\section{INTRODUCTION}

Contractors usually relay on their experience and intuitively make the bidding decisions. However, such practice does not guarantee consistent outcomes. Thus, a structured framework for making the bidding decisions can be of great help especially to new contractors who do not have considerable experience in dealing with complex bidding situations.

Recently, there has been a great interest in the implementation of expert systems (ES) and artificial neural network (ANN) techniques on various areas in the construction industry including the bidding process. The ES technology incorporates decisionsupport models based on heuristic if-then rules elicited from human experts. ANNs are defined as a type of information processing system whose architecture is inspired by the structure of the human brain [1]. Multi-layer perceptrons with backpropagation learning algorithm are the most commonly used ANNs. Back-propagation learning algorithm was proposed by Rumelhart et al [2].

The architecture of multi-layer perceptrons consists of three main components:

1. An input layer containing a set of nodes one for each input variable. These nodes do not perform any mathematical calculations. Therefore, sometimes the input layer is refereed to as the input buffer so it can be distinguished from other layers. The inputs received by this layer are forwarded to the next layer without any changes;

2. Processing elements (PEs) organised into a set of hidden layers. Each PE sums up the values received from the previous layer and uses a formula called the transfer function to produce its output, which is forwarded to all the PEs in the next layer;

3. An output layer containing a number of PEs one for each output. These PEs sum the values forwarded by the last hidden layer and apply their transfer function to produce the final outputs; and,

4. Unidirectional weighted connections between adjacent layers. The connection weights are numerical positive or negative values depending on the information being transmitted. It is by adjusting the connection weights that the ANNs learn from examples.

The communication with the outside word is through the input buffer and the output layer. The hidden layers give critical computation ability to the system [3].

Figure 1 illustrates the structure of a perceptron composed of input buffer with (n) nodes, two hidden layers containing 5 and 1 PEs respectively, and an output layer with one PE.

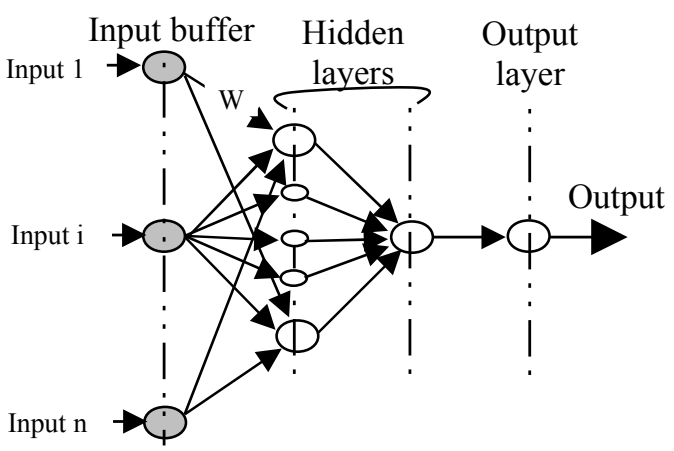

Figure 1. A multi-layer perceptron 
Figure 2 illustrates a processing element with a sigmoid transfer function. Different transfer functions can be used in the same network if required.

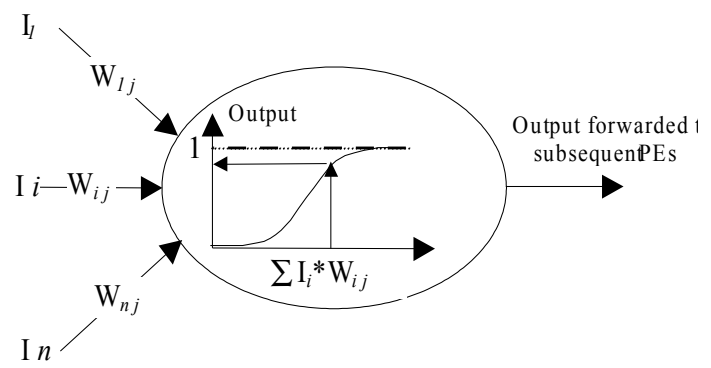

Figure 2. A processing element with a sigmiod transfer function

The development of an ANN application is an iterative trial and error process, which involves many design considerations. These include: modelling the problem under consideration, selection the number of hidden layers and their PEs, and the learning parameters. Some rules of thumb are suggested in the literature to guide this process ([4], [5]).

ANNs have been proposed by many researchers as very reliable tools for modelling unstructured problems including the mark up selection process ([4], [6]).

The present paper investigates the suitability of the ANN techniques to modelling the "bid/ no bid" decision-making process. The following section presents a brief review of the related existing bidding models.

\section{PREVIOUS STUDIES}

Numerous researchers proposed hundreds of bidding strategies, the majority of which are mathematical and statistical models concerned with estimating the probability of winning a contract with a certain mark up. The mathematical complexity of these models made them unpopular in the construction industry ([7],[1]). Recently, the bidding problem has been approached practically rather than mathematically using artificial intelligence techniques such as expert systems (ES) and artificial neural networks (ANN).

However, research continues to focus on the mark up part of the bidding process and neglects the first part although it is at least equally important. Very few publications that address the "bid/ no bid" process can be found in the construction literature.

Ahmad and Minkarah [7] conducted a questionnaire survey to uncover the factors that characterise the bidding decision-making process in the United States. Subsequently, Ahmad [9] proposed a bidding methodology based on the decision analyses technique for dealing with the "bid/ no bid" problem. This model demands many inputs some of which the bidder, especially those with limited experience, might not be able to provide.
Also, it assumes that all factors contribute positively to the "bid" decision. No distinction was made between some factors that count for the "bid" decision, such as profitability, and others that count against it, such as "degree of hazard". However, this approach is the most promising step on the road of modelling the "bid/ no bid" decision.

Shash [10] identified, through a modified version of the same questionnaire used by Ahmad and Minkarah, fifty five factors that characterise the bidding decisions in the UK. The need for work, number of competitors tendering and experience in similar projects were identified as the top three factors that affect the "bid/ no bid" decision.

AbouRizk et al [11] proposed an expert system called BidExpert. This model retrieves historical information from past bids submitted by the company and its competitors. BidExpert uses its knowledge base and provides the user with a "bid/ no bid" recommendation. The necessity for historical information limits the applicability of this model.

Wanous et al [12] conducted a questionnaire survey among Syrian general contractors to uncover the factors that characterise their "bid/ no bid" decision-making process. Thirty eight factors were ranked according to their relative importance in making the "bid/ no bid" decision in Syria.

Subsequently, Wanous et al [13] considered the most important factors and developed a parametric profile each one. All a contractor needs when using this model is his/ her subjective assessments of the considered bidding situation in terms of certain criteria. The contractor's assessment of a certain factor is compared with its parameters to quantify the contribution of this factor in the final recommendation. Only when the accumulated contribution of all factors is positive, will a "bid" recommendation be made with an associated degree of confidence. This model was tested on twenty real bidding situations and succeeded to simulate the actual decisions of $85 \%$ of them.

Dawood [1] used expert systems to help in making the "bid / no bid" decision in the make-to-order precast industry. The explicit knowledge representation and the explanation facility are the main advantages of the ES. However, the practicality of applying this technique can be questioned because it is extremely difficult to explain the process of making the "bid/ no bid" decision through if-then rules [9].

\section{THE DEVELOPMENT PROCESS}

The development framework used in this paper can be divided into the following steps as illustrated in figure 1:

- Data elicitation and analysis;

- Initial design;

- Training;

- Testing; and,

- Adjustment; 
These steps are explained in the following sections.

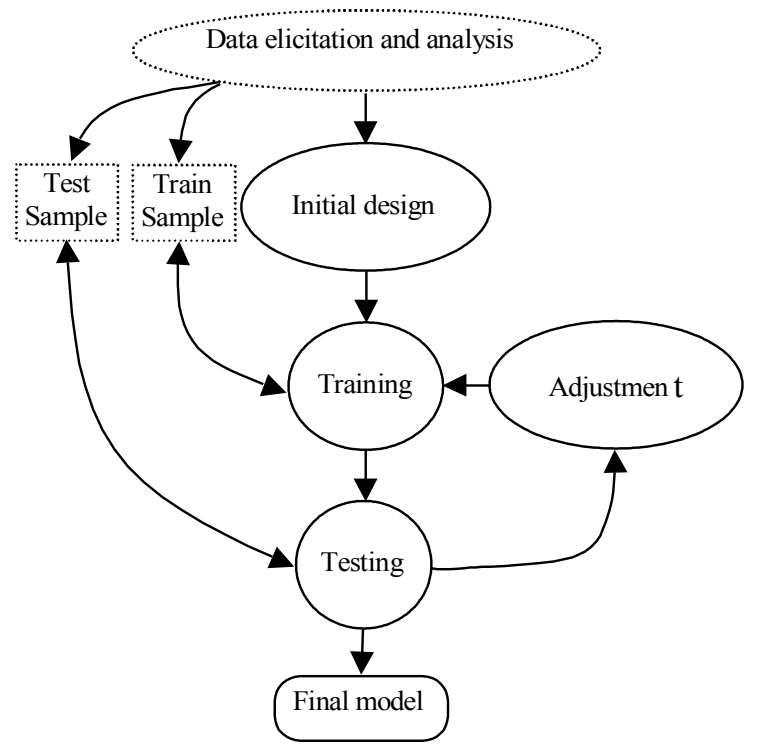

Figure 3. Framework for developing ANN models

\subsection{Data elicitation and analysis}

The most important twenty two bidding factors identified by Wanous et al 1998 were using in designing a simple form to collect situation-outcome data on real bidding situations. The considered factors were listed in the form each with a scale from 0 to 6 where 0 represents "extremely low" and 6 represents "extremely high". Three hundred copies were sent to 30 Syrian general contractors (ten copies each). Respondents were requested to fill in a form for each bidding situation they deal with by providing their actual bid/ no bid decision and their subjective assessments of the considered bidding situation in terms of the listed factor. One hundred and eighty two forms were filled in the returned (60\% response rate). The actual decisions were replaced by numerical values; "bid" with one and "no bid" with zero. Twenty cases were randomly selected and reserved for the validation process. A detailed statistical analysis was made on the remaining one hundred and sixty two cases. The cause-effect relationships between the bidding factors and the actual bid/ no bid decisions were studied through a simple correlation analysis. Factors whose correlation coefficients are greater than 0.40 were selected. The Remaining ones were omitted. Table 1 shows the considered twelve factors with their Pearson correlation coefficients (r).

\subsection{Initial design decisions}

The factors shown in Table 1 were considered as the input variables of the initial ANN bid/ no bid model (called M1). The simplest topology was adopted for this model as a starting point. The input buffer contained twelve nodes fully connected to the output
Table 1. The most influential "bid/ no bid" factors

\begin{tabular}{lcc}
\hline \multicolumn{1}{c}{ Factors } & $\mathrm{r}$ & $|\mathrm{r}|$ \\
\hline 1. Fulfilling the to-tender conditions & +0.69 & 0.69 \\
2. Site accessibility & +0.64 & 0.64 \\
3. Site clearance of obstructions & +0.57 & 0.57 \\
4. Availability of capital required & +0.52 & 0.52 \\
5. Availability of materials required & +0.51 & 0.51 \\
6. Proportions that could be & & \\
$\quad$ constructed mechanically & +0.49 & 0.49 \\
7. Confidence in the cost estimate & +0.46 & 0.46 \\
8. Financial capability of the client & +0.44 & 0.44 \\
9. Public objection & -0.43 & 0.43 \\
10. Workload & -0.42 & 0.42 \\
11. Reputation of the client & +0.42 & 0.42 \\
12. Favourability of the cash flow & +0.41 & 0.41 \\
\hline
\end{tabular}

layer, which contains only one processing element (PE) for the only output. This output is called the Neural Bidding Index (NBI). The model will make the "bid" recommendation when NBI is greater than $(0.5)$. The closer the value of NBI is to one the more confidence in the "bid" recommendation and the closer it is to zero, the more confidence in the "no bid" recommendation. The "normalised cumulative delta" learning rule and the sigmoid transfer function were used. The other parameters were set to their default values selected by the used development software (NeuralWorks) [14]. The initial weights were automatically set to random small numbers between $(-0.5)$ and $(+0.5)$.

\subsection{Training}

The back propagation learning algorithm was used to modify initial connection weights. A fixed number of training iterations (50000) was used in this stage. When the learning counter reaches this limit, the learning was automatically ceased. The ability of mode (M1) to explain the variance in the training data after 50000 iterations was presented by its training diagnostic measurements $(\mathrm{RMS} \mathrm{train}=0.1022$ and $\left.\mathrm{R}^{2}{ }_{\text {train }}=0.8491\right)$. The generalisation ability of $\mathrm{M} 1$ after

training is tested in the following subsection.

\subsection{Testing}

The projects reserved for the validation process were used to examine the generalisation capability of model (M1) after training. The contractors' assessments of these situations were presented to model (M1). The produced outputs were compared to the actual ones and the used software provided two measures of the test result. These measures (RMS test $=0.1658$ and $\mathrm{R}^{2}{ }_{\text {test }}=0.7983$ ).

\subsection{Adjustment}

In this stage the initial model was modified, i.e. fine tuned. There are endless possibilities of how the model can be modified. These include:

1. Adding hidden layer(s) and experimenting with different numbers of processing elements; 
2. Examining different learning rules such as the delta rule and the cumulative delta rule;

3. Examining different transfer functions such Tangent and linear functions; and,

4. More training iterations.

After examining 57 different models, the training and testing diagnostics ( $\mathrm{R}^{2}$ and $\mathrm{RMS}$ ) were improved considerably $\left(\mathrm{RMS} \mathrm{S}_{\text {train }}=0.0112, \mathrm{R}_{\text {train }}=0.9999\right.$ and RMS ${ }_{\text {test }}=0.1522, \mathrm{R}^{2}$ test $\left.=0.8484\right)$. The structure of the corresponding model is composed of an input buffer with twelve nodes, two hidden layers with five PEs in the first one and one PE in the other, and an output layer with one PE (see Figure 1). This model was selected as the best model.

\section{VALIDATION}

In order to be accepted as a decision-support tool, the model needs to be validated. Therefore, the developed bid/ no bid model was used to predict the actual decisions of the twenty projects included in the test sample. The actual decisions were successfully predicted in eighteen cases, which means that the model is $90 \%$ accurate in simulating the actual decisions of the test samples that have not been used in the training process. This result is very encouraging and leading to the conclusion that the ANNs technology is a suitable tool for modelling the bid/ no bid decision-making process.

\section{CONCLUSION}

This paper reviewed the "bid/ no bid" models available in the current literature and concluded that this decision has received little attention from researchers compared with the mark up part of the bidding process. The applicability of the artificial neural networks on the bid/ no bid process was investigated. Data on one hundred and sixty real-life construction projects was used to develop an innovative bid/ no bid model. The developed model was validated using another twenty real projects. It proved to be $90 \%$ accurate in simulating the actual decisions of these projects. That means the model is more accurate than a parametric model proposed in previous work [13] (see section 2). This result provides evidence that the ANN technology can be applied to the bid/ no bid process with high confidence. Although the developed model is based on data on projects from the Syrian construction industry, it provides a universal "shell" that can be applied in other countries.

\section{REFERENCES}

[1] Dawood, N. N. An integrated bidding management expert system for the make-to-order precast industry. Construction Management and Economics, Vol.13, No. 2, pp. 115-125, 1995.
[2] Rummelhart, D.E., Hinton, G.E., and Williams, R.J. Learning representation by back propagation error. Nature, Vol. 323, pp. 533-536, 1986.

[3] Boussabaine, A.H., Thomas, R. and Elhag, T.M.S. Modeling cost-flow forecasting for water pipeline projects using neural networks. Journal of Engineering, Construction and Architectural Management. Vol.6, No. 3, pp 213-224, 1999.

[4] Boussabaine, A.H. The use of artificial neural networks in construction management. Construction Management and Economics. Vol.14, pp 427-436, 1996.

[5] Hegazy, T., Fazio, P. and Moselhi, O. Developing practical neural network applications using BackPropagation. Microcomputers in Civil Engineering, Vol.9, pp 145-159, 1994.

[5] 1- Moselhi, O., Hegazy, T., and Fazio, P. Neural net works as tools in Construction. ASCE journal of Engineering and Management. 117, 4, pp. 606-625, 1991.

[7] Ahmad, I., and Minkarah, I. A. Questionnaire survey on bidding on construction. ASCE Journal of Management in Engineering, Vol. 4, No. 3, pp. 229243, 1988.

[8] Moselhi, O., Hegazy, T. and Fazio, P. DBID: Analogy Based DSS for Bidding in Construction. ASCE Journal of Engineering and Management. 119, 3, pp. 466-470, 1993.

[9] Ahmad, I. Decision- support system for modelling the bid/ no bid decision problem. Journal of construction engineering and management. Vol. 116, No. 4, pp 595-607, 1990.

[10] Shash, A. Factors considered in tendering decisions by top UKcontractors. Construction Management and Economics, Vol.11, No.2, pp.111118, 1993.

[11] AbouRizk, S.M., Dozzi, S.P. and Sawhney, A. BidExpert- An Expert System for Strategic Bidding. Annual conference of the Canadian Society of Civil Engineering, Fredericton, NB, Canada. Pp. 39-48, 1993.

[12] Wanous, M., Boussabaine, A.H. and Lewis, J. Tendering factors considered by Syrian contractors. ARCOM, $14^{\text {th }}$ annual conference proceedings, Vol. 2, pp. 535-534, Oxford, England, 1998.

[13] Wanous, M., Boussabaine, A.H. and Lewis, J. $\mathrm{Bid} /$ no bid: a parametric solution.C onstruction Management and Economics. Vol.18, No.4, pp.457466, 2000.

[14] Neural Computing. A technology handbook for Professinal II/PLUS and NeuralWorks Explorer. NeuralWare, Inc., 1996. 